\title{
Cinémas
}

Revue d'études cinématographiques

Journal of Film Studies

\section{Spectacles douloureux, exhibitions malsaines. Présentations et représentations de la mort à Paris au XIX ${ }^{\mathrm{e}}$ siècle Painful Spectacles, Unhealthy Displays: The Presentation and Representation of Death in Nineteenth-Century Paris}

\section{Patrick Désile}

Volume 20, numéro 2-3, printemps 2010

L'horreur au cinéma

URI : https://id.erudit.org/iderudit/045144ar

DOI : https://doi.org/10.7202/045144ar

Aller au sommaire du numéro

Éditeur(s)

Cinémas

ISSN

1181-6945 (imprimé)

1705-6500 (numérique)

Découvrir la revue

Citer cet article

Désile, P. (2010). Spectacles douloureux, exhibitions malsaines. Présentations et représentations de la mort à Paris au XIX ${ }^{\mathrm{e}}$ siècle. Cinémas, 20(2-3), 41-63. https://doi.org/10.7202/045144ar
Résumé de l'article

Tenant pour acquis que le thème central du cinéma d'horreur est la mort, l'auteur s'interroge sur le contexte de son émergence en examinant (dans les limites du domaine français) des spectacles qui lui sont antérieurs et dont la mort était l'objet. Le XIX ${ }^{\mathrm{e}}$ siècle a connu, à côté d'innombrables représentations, des spectacles de présentation de la mort. L'exposition publique à la Morgue et les exécutions capitales étaient offertes à une large fréquentation ; des récits accompagnaient ces visions. Mais le cinéma d'horreur ne constitue pas pour autant leur prolongement par d'autres moyens. L'auteur expose en effet un processus plus complexe. Le regard sur la mort change au cours du siècle. À partir des années 1870 s'amorce un processus d'occultation. L'exposition à la Morgue cesse en 1907 ; l'abolition de la peine de mort est envisagée en 1908. Pourtant, dans le même temps, le cinéma montre des scènes d'exécutions. Quand les décapitations, un temps suspendues, reprennent spectaculairement en 1909, elles sont, semble-t-il, filmées. Mais toute projection de scène d'exécution est aussitôt interdite : c'est la naissance de la censure cinématographique en France. Le spectacle de la décapitation réelle est dès lors tabou au cinéma. C'est qu'il peut être considéré comme spectacle de l'horreur par excellence, pour plusieurs raisons que l'examen de l'imaginaire de la décapitation fait apparaître. 


\section{Spectacles douloureux, exhibitions malsaines. Présentations et représentations de la mort à Paris au XIX ${ }^{\mathrm{e}}$ siècle}

\section{Patrick Désile}

\section{RÉSUMÉ}

Tenant pour acquis que le thème central du cinéma d'horreur est la mort, l'auteur s'interroge sur le contexte de son émergence en examinant (dans les limites du domaine français) des spectacles qui lui sont antérieurs et dont la mort était l'objet. Le XIX siècle a connu, à côté d'innombrables représentations, des spectacles de présentation de la mort. L'exposition publique à la Morgue et les exécutions capitales étaient offertes à une large fréquentation; des récits accompagnaient ces visions. Mais le cinéma d'horreur ne constitue pas pour autant leur prolongement par d'autres moyens. Lauteur expose en effet un processus plus complexe. Le regard sur la mort change au cours du siècle. À partir des années 1870 s'amorce un processus d'occultation. L'exposition à la Morgue cesse en 1907; l'abolition de la peine de mort est envisagée en 1908. Pourtant, dans le même temps, le cinéma montre des scènes d'exécutions. Quand les décapitations, un temps suspendues, reprennent spectaculairement en 1909, elles sont, semble-t-il, filmées. Mais toute projection de scène d'exécution est aussitôt interdite: c'est la naissance de la censure cinématographique en France. Le spectacle de la décapitation réelle est dès lors tabou au cinéma. C'est qu'il peut être considéré comme spectacle de l'horreur par excellence, pour plusieurs raisons que l'examen de l'imaginaire de la décapitation fait apparaitre.

\section{For English abstract, see end of article}

Est-ce bien le nom de curiosité qu'il faut donner à cette force qui nous attire vers les spectacles douloureux et les exhibitions malsaines, vers le mystérieux et le tragique?

Émile Deschamps (1897, p. 165) 
L'horreur cinématographique commencerait avec Mario Bava, ou bien avec Murnau ou Robert Wiene, à moins que ce ne soit avec Georges Méliès. Quelques auteurs citent même des films de 1893, voire de 1889 ! Mais pourquoi ne pas remonter au néolithique, si l'horreur est simplement humaine? Plus légitimement, on invoque le précédent des fantasmagories de Robertson, on effectue un parallèle avec le théâtre du Grand-Guignol. Tout de même, il semble que, dès lors qu'on s'interroge sur les prémices de l'horreur cinématographique, un peu de confusion règne, et une certaine perplexité se fait jour.

Lobjet du présent article n'est pas de rechercher quelques nouveaux "précurseurs", ou quelque origine un peu sûre: de telles quêtes sont évidemment vaines, sans logique et sans fin; je ne prétends pas non plus tout clarifier d'une question si difficile; je voudrais seulement évoquer quelques aspects du contexte de l'émergence d'un cinéma "d'horreur ", en le rapprochant d'autres pratiques, en particulier d'autres pratiques de spectacles, et en me limitant, d'ailleurs, au domaine français, particulièrement à la situation parisienne, au XIX ${ }^{\mathrm{e}}$ siècle et durant les premières années $\mathrm{du} \mathrm{XX}^{\mathrm{e}}$. Situation singulière, sans doute, mais dont l'analyse me semble, à bien des égards, éclairante.

Le premier point sur lequel achoppe la réflexion, c'est évidemment la question de l'horreur elle-même. Il peut être légitime, au moins dans une première approche, de se pencher sur un objet défini par l'effet qu'il est censé produire ou, du moins, par le projet de le susciter. Cela soulève évidemment des questions, et d'abord celle de la définition de cet effet. On admettra difficilement que le cinéma d'" horreur" recherche simplement ce "violent saisissement d'effroi» (pour citer le Trésor de la langue française) que l'horreur est censée provoquer, et l'on peut supposer, en l'occurrence, que l'on n'a pas affaire à un jeu de stimulus-réponse, mais plutôt à un jeu subtil, ambigu, plein de faux-semblants autour de l' ' horreur $^{1} "$.

En tout état de cause, cette approche n’a de sens que par référence à un genre déjà constitué, autour de ce jeu, précisément. Rien n'autorise à la transposer à d'autres spectacles, qui ne présument pas, fût-ce malicieusement, la production d'effets déterminés. On sait bien d'ailleurs que l'une des difficultés de 
l'histoire culturelle, particulièrement de l'histoire des spectacles, réside dans le manque de données sur les publics, presque toujours plongés dans une ombre que peu de sources viennent dissiper. S'il est malaisé de savoir simplement qui sont les spectateurs, ce qu'ils disent ou ce qu'ils font, savoir ce qu'ils ressentent est à peu près impossible.

Sans doute y eut-il des spectacles dont les promoteurs cherchaient à susciter quelque chose comme un sentiment d'horreur chez les spectateurs; quelques témoignages laissent supposer qu'ils y parvenaient, ou du moins (car encore une fois, ce que ressentaient les spectateurs nous est inconnu) qu'une sorte de jeu, dont il faudrait là aussi préciser la nature, s'instaurait autour de l'horreur. Les fantasmagories de Robertson et les spectacles du GrandGuignol relèvent de cette catégorie. Est-ce à dire que la réflexion sur l'émergence de l'horreur cinématographique doive se borner à ces quelques rapprochements? Non, sans doute: l'examen de spectacles considérés isolément, et choisis en fonction d'un critère qui n'est pas clarifié, l'effet qu'ils sont supposés produire, ne saurait être un point de départ satisfaisant. Cette question de l'effet doit donc, au moins provisoirement, être laissée en suspens.

Mais si l'on admet que l'horreur cinématographique ne peut être dissociée du thème de la mort, il est légitime de s'attacher aux spectacles dont la mort est, pendant la période qui précède l'émergence du cinéma, l'objet principal ou le thème essentiel, et de voir comment le cinéma s'insère parmi eux, ce qu'il continue ou ce qu'il rend périmé.

Or la mort, on le sait, hante tout le XIX siècle, et même un long XIX siècle qui court des dernières décennies du siècle précédent aux premières années du suivant. C'est l'époque du roman gothique, $\mathrm{du}$ romantisme noir, de Baudelaire et, à la fin du siècle, du symbolisme, du décadentisme et de leur esthétique du macabre. C'est Géricault peignant des têtes coupées, des membres épars. C'est Berlioz se faisant ouvrir, à Florence, le cercueil d'une jeune morte et résistant à la tentation de l'embrasser (Vovelle 1983, p. 584). C'est, en 1829, Lầne mort et la femme guillotinée, roman de Jules Janin qui fit date et fut souvent réédité, où sont décrits successivement la mise à mort d'un âne par des chiens, la Morgue, l'hôpital, la prison et l'échafaud, le cimetière de Clamart et, lieu de l'ultime 
avilissement d'un corps, l'amphithéâtre de dissection. C'est encore la poésie fin de siècle et nécrophile de Maurice Rollinat (1885, p. 387), dont L'épitaphe, par exemple, se conclut par «Vive la mort! Vive la mort!». Et c'est tout un imaginaire de fantômes, de mortsvivants, de larves et de goules que la culture urbaine reprend à une paysannerie qui commence à le délaisser. Car cette culture de la mort se déploie sur deux registres: le cadavre, mais lâme, les esprits errants, mais la putréfaction des chairs.

Et l'on sait bien aussi que tout cela n'intéresse pas la seule culture des élites: la mort est au cœur de bien des productions de la culture de masse naissante, mélodrames, romansfeuilletons, faits divers des journaux, et toute une imagerie. À la fin du siècle, au cabaret du Néant du boulevard de Clichy, le lustre est un squelette et les tables sont des cercueils.

Tout cela, ce sont des représentations de la mort. Mais le XIX siècle connaît aussi des spectacles de présentation de la mort et du cadavre.

Il y a, d'abord, des spectacles de la mort animale. Jusqu'au milieu du XIX siècle au moins, subsistent, malgré les interdictions, de ces combats d'animaux que le XVIII ${ }^{e}$ siècle avait prisés. C'est l'un d'eux, un peccata (âne contre chiens), que décrit Jules Janin (1842, p. 8). Un âne boiteux entre dans l'arène suivi de quatre dogues: "Ils déchirent son corps en lambeaux; ils le percent de leurs dents aiguës $[\ldots]$. Bientôt le sang coule $[\ldots]$. Enfin l'âne tombe sous leurs dents.»

Mais il y a surtout des spectacles, qui ne sont certes pas de divertissement, de la mort et du cadavre humains. Dans les premières décennies du XIX ${ }^{\mathrm{e}}$ siècle, les dissections, par exemple, ne sont pas confinées dans le secret de l'hôpital. "Rien de plus horriblement infect que ces amphithéâtres privés, situés sous les combles des plus misérables maisons des rues étroites et fangeuses que renfermait, il y a quelques années encore, le quartier latin ", lit-on, en 1869, dans le dictionnaire de Dechambre (1869, p. 752). Encore peut-on penser que, dans ce cas, le public est majoritairement composé d'initiés.

Tel n'est pas le cas de deux spectacles en quelque sorte institutionnels et offerts à la plus large fréquentation: l'exposition publique de cadavres de la Morgue et les exécutions capitales. 
La Morgue existe à la fin de l'Ancien Régime. Elle est installée dans une ancienne geôle du Châtelet; on y présente des cadavres inconnus; mais le lieu est exigu et mal éclairé. Dès la fin du $\mathrm{XVIII}^{\mathrm{e}}$ siècle, on conçoit le projet d'un établissement où les corps seraient bien visibles. Il aboutit en 1804. La Morgue est implantée au cœur de Paris, sur l'île de la Cité. Les cadavres, ceux des noyés, des morts inconnus ramassés dans la rue y sont exposés, allongés sur des tables de marbre, la tête un peu surélevée, derrière une large vitrine, sous un éclairage zénithal. La justification de ce dispositif, c'est la nécessité d'identifier les corps. Aussi la Morgue est-elle ouverte à tous, tous les jours de l'année, toute la journée. Le public est même incité à s'y rendre. Et pendant tout le XIX ${ }^{\mathrm{e}}$ siècle, la Morgue est un spectacle. "La foule, qui s'écrase à certains jours devant les vitrines de la salle d'exposition, n'y vient chercher que des émotions violentes; ce n'est pour elle qu'un spectacle à sensation, permanent et gratuit" (Cherbuliez 1891, p. 344). Dans quelques cas, les corps sont même apprêtés et mis en scène: une petite fille, embaumée, est présentée assise en 1877, une autre en 1886 (Bertherat 2003, p. 197).

Dès l'origine, mais particulièrement dans les dernières décennies du XIX ${ }^{\mathrm{e}}$ siècle, certains cadavres (car la Morgue en reçoit plusieurs centaines par $a^{2}{ }^{2}$ ) sont pris dans une sorte de récit collectif où se mêlent du réel et de l'imaginaire: la rumeur publique, mais surtout la grande presse populaire en font des personnages à demi fictifs. Le succès est constant tout au long du siècle, et l'affluence, pour certaines affaires, est considérable, comme pour la femme coupée en morceaux de 1876 ou pour la petite fille de la rue du Vert-Bois en 1886 (Bertherat 2003, p. 250).

Le succès public des exécutions capitales n'est pas moindre et les comptes rendus dont elles font l'objet dans la presse manquent rarement de souligner le grand nombre des spectateurs et leur impatience. Ces récits constituent une sorte de genre journalistique, assez stéréotypé et généralement sobre, mais glaçant. Ils sont d'ailleurs eux-mêmes l'épilogue de longs récits à épisodes, surtout dans la deuxième moitié du XIX siècle, qui comportent la description de la scène du crime, le compte rendu de l'enquête et celui du procès. 
Mais les exécutions ne font pas seulement l'objet d'articles de presse: la guillotine est un motif littéraire et iconographique récurrent, presque obsédant ${ }^{3}$. L'une des raisons de cette obsession, c'est la fascination pour l'instantanéité de la mort, de la section de la tête, de la séparation, fulgurante, de l'âme et du corps. De là découlent bien des angoisses et bien des récits: on a pu croire, et ce dès le XVIII ${ }^{\mathrm{e}}$ siècle, que la tête continuait à sentir quelque temps, et que l'individu décapité assistait donc, comble, peut-être, de l'horreur, à sa propre mort. Le thème court tout au long du $\mathrm{XIX}^{\mathrm{e}}$ siècle et inspire des récits de morts-vivants modernes. Ainsi en est-il de l'histoire de la femme au collier de velours, contée par Washington Irving (1951 [1824]), adaptée par Petrus Borel (1843), reprise par Alexandre Dumas (2006 [1849]) et même transposée, tardivement, par Gaston Leroux (1924): pendant la Révolution, un homme rencontre une femme, passe la nuit avec elle; au matin, quand son collier de velours est ôté, "cessant d'être maintenue par le seul lien qui la rattachait aux épaules, la tête de la suppliciée roul[e] du lit à terre» (Dumas 2006, p. 229).

Si la figure de la guillotine et celle de la tête coupée obsèdent tout le XIX ${ }^{e}$ siècle, c'est aussi pour d'autres raisons, nombreuses, entrelacées, changeantes. Nous reviendrons plus loin sur cette question essentielle, sans prétendre faire plus que l'effleurer.

D'un bout du siècle à l'autre, donc, les écrivains et les journalistes ressassent des récits ambigus et morbides, les foules voyeuses se pressent vers les cadavres et vers les échafauds: comment les images et les récits que le cinéma, né de ce siècle même, adresse aux foules, ne prolongeraient-ils pas ces fascinations et ces obsessions? Le cinéma continuerait, en somme, à faire vivre ces visions du XIX ${ }^{e}$ siècle avec ses propres moyens.

Cela n'est pas tout à fait faux, et le transfert d'une imagerie n'est pas inconcevable, mais les choses sont plus complexes. Le $\mathrm{XIX}^{e}$ siècle est de bout en bout obsédé par la mort, sans doute, mais ce n'est pas toujours le même regard qu'il porte sur elle. On peut distinguer au moins deux moments, dont le second commencerait, grossièrement, vers 1870 .

Si les écrivains romantiques parlent tant de la mort, c'est aussi, simplement, trivialement, que, comme leurs lecteurs et comme tout le monde, ils la croisent et la fréquentent commu- 
nément. Les souvenirs de la Terreur, ceux des massacres de Septembre ou des guerres de l'Empire sont présents à tous les esprits et émaillent les conversations. Et cette société reste très violente: pendant plus d'un demi-siècle, la guerre civile est endémique, et l'on meurt sur les barricades.

On est, par ailleurs, démuni face à la maladie. La médecine engrange des savoirs, mais ses moyens thérapeutiques restent limités. Les épidémies sont dévastatrices. Le choléra, qui survient à Paris en 1832, fait plusieurs milliers de morts. Il frappe encore en 1849 et en 1865 , à peine moins meurtrier.

Heine (1878, p. 148) décrit son irruption le 29 mars 1832, en plein carnaval, les masques qu'on enterre à la hâte dans leurs costumes de bal: "De quelque côté qu'on regardât dans les rues, on ne voyait que convois funèbres..." Et d'autres morts, bientôt, s'ajoutent aux cholériques: des hommes sont massacrés par la foule qui les croit empoisonneurs, comme celui dont Heine découvre, rue de Vaugirard, le corps nu, déchiré, sanglant... (p. 140). De telles scènes de violence ostensible se produisent ici et là dans la première moitié du XIX ${ }^{\mathrm{e}}$ siècle.

La violence institutionnelle n'est pas davantage dissimulée, la publicité est inscrite dans la loi. Les exécutions capitales ont lieu en pleine ville, en plein jour, sur l'échafaud qui les rend encore plus visibles; et elles ne sont pas rares: 72 condamnations à mort exécutées par an, en moyenne, en France, entre 1826 et 1830 . Le nombre décroît ensuite, mais se maintient à une trentaine par an jusqu'en 1860 (Anonyme 1989, p. CXL). Et, pour beaucoup, voir guillotiner est une espèce de fête. Dans la saynète L'exécution (1829), Henry Monnier, dont les contemporains comparaient les dialogues à des daguerréotypes, met en scène deux gamins, Lolo et Titi ; "Viens-tu voir guillotiner?" demande Lolo, qui ajoute: "Nous irons à Clamart.» "Quoi faire?" demande Titi. "Pour tout voir jusqu'à la fin; c'est là qu'on vide les paniers" (1984, p. 91). Lolo s'y entend, son père a vu jusqu'à soixante exécutions par jour, "qu'les ruisseaux en étaient tout rouges» (p. 93). En place de Grève, des loueurs de places circulent dans la foule des habitués. Mais Lolo et Titi se juchent sur un réverbère, puis commentent, goguenards, chaque instant des exécutions. 
Et Flaubert (1980, p. 321) se souvient d'avoir vu, enfant, à six ou sept ans, comme il rentrait de l'école en passant par la place du Vieux-Marché, à Rouen, la guillotine, qui venait de servir. "Il y avait du sang frais sur les pavés, et on défaisait le panier.» [Lettre à Louise Colet, 30 avril- $1^{\text {er }}$ mai 1853].

Quant aux cadavres de la Morgue, quelle que soit leur hideur, ils sont, jusqu'en 1877, exhibés nus (Bertherat 2003, p. 174 sqq.). Mais la vision de la nudité des cadavres n'est pas, sans doute, bien exceptionnelle: l'usage s'est longtemps maintenu, en tout cas à la campagne, d'enterrer le corps nu dans le linceul (Van Gennep 1998, p. 609).

Il y a donc cette visibilité familière de la mort, du cadavre et du sang. Mais dans les dernières décennies du siècle, pendant cette période qui se soucie tant, par ailleurs, d'animer les photographies, la mort recule et le cadavre se dérobe.

La mort recule: la révolution pastorienne, les progrès de l'antisepsie font chuter plus nettement les taux de mortalité, mais surtout attestent l'efficacité d'une médecine qui identifie les agents pathogènes et dont on peut désormais beaucoup espérer. C'en est d'ailleurs fini, du moins pour ce siècle-là, des grandes épidémies cauchemardesques. C'en est fini aussi, après la Commune, des grandes insurrections meurtrières.

Dans le même temps, on cherche à dérober les cadavres au regard. On a vu quel dégoût inspirait en 1869 à l'auteur de l'article "Amphithéâtre" du dictionnaire de Dechambre ces dissections abjectes pratiquées au cœur de la ville. Aussi voulaitil que les établissements qui les pratiquaient fussent éloignés des habitations, à l'abri de hauts murs aux ouvertures occultées (p. 753). Ce souci d' «éviction» et de dissimulation de la mort et des cadavres est général et constant, et revêt de nombreux aspects.

L'habitude se répand partout d'habiller, avant de les porter en terre, des cadavres qui n'ont plus rien, dès lors, de charognes; ils sont en somme d'anciens vivants, inanimés, mais encore socialisés.

On tend aussi à éloigner les cimetières des églises, du centre des villes et des villages; pour Paris, Haussmann juge les grands cimetières périphériques encore trop visibles et conçoit, en 1864, une nécropole qui regrouperait tous les cimetières 
parisiens en pleine campagne, à Méry-sur-Oise, et à laquelle on accéderait par le train (Ariès 1977, p. 248).

Tout se passe donc comme s'il s'agissait vraiment de mettre la mort hors de vue. À tout le moins, on dressera devant elle l'écran d'une esthétique. C'est dans la deuxième moitié du $\mathrm{XIX}^{\mathrm{e}}$ siècle que prolifere une architecture mortuaire de chapelles et de monuments, que surgit une foisonnante statuaire funéraire, qu'on prend l'habitude de fleurir les tombes de chrysanthèmes (Van Gennep 1998, p. 657). C'est alors aussi qu'apparaît, puis que s'élargit, par degrés, le liseré noir des faire-part: d'un mince filet qu'il était vers 1850, il devient une large bordure à la fin du siècle (Vovelle 1983, p. 629), comme s'il s'agissait, à travers cette insistance croissante sur ce signe graphique de la mort, austère et pur, de détourner pudiquement le regard du cadavre pourrissant. Le refus de l'envisager semble attesté par l'apparition d'une autre pratique: la crémation, autorisée à Paris en 1880 et légalisée en 1889 (Vovelle 1983, p. 657). Elle reste marginale, mais dessine pourtant un désir de dématérialisation, d'invisibilité des corps morts.

On discerne aussi, d'autre part, à la fin du siècle surtout, une aspiration à la croyance en la désincarnation des morts. On veut croire, en effet, malgré la science ou avec elle, et en marge du christianisme, à leur survie incorporelle. C'est la vogue du spiritisme, pour lequel le corps n'est que le lieu temporaire d'incarnation d'un esprit qui survit donc après le décès. Les personnalités les plus positives s'interrogent sur cette incorporéité des êtres. Charles Richet, prix Nobel de médecine en 1913, croit ainsi fermement aux fantômes (Edelman 2006, p. 125). Ce sont encore des manières d'occulter la matérialité visible de la mort.

Dans ce contexte, on conçoit que l'étalage de chairs livides de la Morgue, que les sanglantes décapitations publiques commencent à détonner et fassent l'objet de débats.

On avait partout vêtu les morts, on rhabille enfin ceux de la Morgue en 1877 (Bertherat 2003, p. 177). La raison invoquée est étrangement celle-là même qui justifiait qu'on les laissât nus : faciliter la reconnaissance. Ce qu'atteste surtout cette décision, sans doute, c'est un changement de regard sur le corps mort, dont on semble vouloir qu'il conserve quelque chose de sa pudeur de vivant. 
À partir des années 1880 , et surtout à partir de la publication, par le magistrat Adolphe Guillot, de Paris qui souffre (1887), des voix s'élèvent pour que l'exposition des corps, considérée comme immorale, obscène, inutile, soit supprimée. Malgré la foule qui afflue toujours, malgré Le Petit Journal qui voudrait son maintien, elle l'est définitivement en 1907.

La guillotine connaît de même un lent processus d'occultation: à Paris, elle quitte la place de Grève, centrale, pour la barrière Saint-Jacques, d'abord, en 1832, puis, en 1851, pour la place de la Roquette, non loin de la prison du même nom; à partir de 1878, elle s'élève près du mur d'enceinte de la prison de la Santé et l'on tient la foule à distance. Depuis longtemps, elle ne fonctionne plus qu'au petit jour. En 1870, on supprime l'échafaud (Corbin 2005, p. 234).

L'ostentation des exécutions est donc en recul constant, mais leur nombre va de toute façon diminuant. De 1876 à 1880 , six exécutions par an en moyenne pour la France; quatre exécutions en 1897 (Anonyme 1900, p. 26), trois en 1901 (Anonyme 1903, p. 26), aucune en 1906 (Anonyme 1908, p. 26). Les présidents Félix Faure et Émile Loubet accordent volontiers la grâce; Armand Fallières, élu en 1906, le fait systématiquement au début de son septennat. Et la peine capitale est tout près de disparaître du code pénal. Une campagne en faveur de son abolition s'organise en effet. Le conseil municipal de Paris, par exemple, le 30 juin 1902, considérant notamment que "la publicité donnée aux exécutions capitales est immorale, corruptrice et dangereuse» (Anonyme 1903a, p. 506), émet le vœu que la peine de mort soit abolie, et, en attendant, que les exécutions aient lieu à l'intérieur des prisons, loin du regard des foules. Les crédits alloués au bourreau ont déjà été supprimés quand, en 1908, Aristide Briand soumet aux députés un projet de loi d'abolition de la peine de mort, qui est cependant rejeté. On va le voir, dans le processus général d'occultation de la mort, la décapitation occupe une place singulière.

La raison immédiate pour laquelle les députés rejettent l'abolition, c'est qu'ils n'ont garde de heurter une opinion publique qui lui est très hostile. Le crime particulièrement odieux commis en 1907 par le violeur et assassin Soleilland, et 
surtout la grâce que lui a accordée Fallières, ont soulevé l'indignation. Le Petit Parisien s'est lancé dans une campagne en faveur du maintien de la peine de mort et dans l'organisation d'un référendum dont les résultats sont publiés le 5 novembre 1907: un million de lecteurs ont voté pour le maintien, trois cent mille pour l'abolition. Quelque soixante-dix jurys populaires veulent en outre pouvoir continuer à infliger la sanction suprême et ont adressé des pétitions en ce sens au gouvernement (Imbert 1998, p. 86).

Ce n'est là, sans doute, qu'un des aspects des résistances au processus général d'occultation de la mort que j'ai décrit. L'abandon de la "sépulture à nu» a suscité des réticences, l'éloignement des cimetières a provoqué des protestations, notamment de la part de l'Église. Le projet d'Haussmann n'a pas abouti et n'a jamais été repris. L'interdiction de l'exposition publique à la Morgue a finalement été décidée, mais non sans avoir rencontré une certaine hostilité : certains, qui consentent à reconnaître que ce spectacle est "aussi impropre que possible à développer de nobles instincts et même de bons sentiments dans la foule» (Cherbuliez 1891, p. 358), jugent néanmoins nécessaire son maintien.

Mais enfin la visibilité de la mort s'est considérablement restreinte. On est passé d'un régime de visibilité impudique et immédiate de la mort à un régime de visibilité parcimonieuse, précautionneuse et médiate. Ce passage s'est accompagné de tensions entre une grande partie du public et un certain nombre de représentants des élites républicaines, élus, magistrats, hauts fonctionnaires, mais aussi des écrivains, des journalistes, qui tendaient à promouvoir l'occultation. Les descriptions de la Morgue et les récits d'exécutions incluent presque toujours un jugement condescendant sur les foules avides de spectacles pervers.

Mais c'est à propos de la guillotine que ces tensions sont les plus vives. Bien entendu, ce qui est en jeu, ce n'est pas seulement la question de la visibilité des exécutions, puisque c'est la peine elle-même qu'il s'agit d'abolir. Mais les deux plans ne sont pas alors dissociables. La publicité est en effet au cœur du débat. Pour les uns, son caractère scandaleux fournit un argument 
décisif en faveur de l'abolition des exécutions, pour les autres, elle est nécessaire à la préservation de leur exemplarité. Il est en outre difficile de ne pas faire la part du voyeurisme sadique dans ce grand mouvement d'approbation de la peine de mort par les foules.

Or, cette tension entre le désir de voir la mort, mais plus précisément, ici, de voir la décapitation, et le souci de n'y pas céder, le tout jeune cinéma va s'y trouver pris.

Le public aime les récits d'exécutions, il en aime le spectacle: le premier cinéma lui en montre, ou du moins lui en présente des reconstitutions. On trouve en 1897 au catalogue Pathé une Exécution capitale à Berlin: c'est une décapitation à la hache, à l'issue de laquelle la tête est montrée au public. Les récits de supplices chinois sont prisés: en 1900, Pathé propose Une exécution à Pékin; la tête du condamné est placée sur un poteau et insultée par «la populace». En 1903, une collection de films d'exécutions capitales est inscrite au catalogue: exécutions en France, en Angleterre, en Allemagne, en Espagne, en Chine, et électrocution du meurtrier du président américain McKinley.

Plusieurs films de fiction comportent des scènes d'exécution, en particulier de décapitation par la guillotine. En 1901, Histoire d'un crime, de Ferdinand Zecca, fait date. Inspiré d'une série de tableaux de cire du musée Grévin, il résume un itinéraire criminel. Mais, malgré le titre, la scène du crime, banale (un homme est tué au cours d'un cambriolage), est surtout une introduction au parcours de l'assassin à partir de son arrestation, avec, en particulier, les tableaux de la confrontation avec la victime à la Morgue (censée provoquer les aveux, elle était en effet pratiquée), de la longue toilette du condamné, enfin de l'exécution par la guillotine, à la fin de laquelle on voit, bien distinctement, la tête tomber.

D'autres films intègrent cette scène ultime, comme La vie d'un joueur, de Zecca, encore, en 1903. Le cas des Incendiaires, de Georges Méliès, en 1906, est remarquable. Le film raconte l'histoire d'une bande de chemineaux chauffeurs et pyromanes; l'un d'eux est arrêté, condamné à mort et décapité. Or ce film anticipe la réalité, on le verra. Il est prémonitoire en ce que, tel un rêve, il est accomplissement d'un désir, du désir collectif de 
voir une tête tomber. Le tableau «de l'exécution sur la place de la Roquette est le plus parfait qui ait été fait en ce genre, c'est la réalité même", dit le texte explicatif (Malthête et Mannoni 2008, p. 196). Et pour cette raison, précisément, il est facultatif. Si Histoire d'un crime a pu être projeté amputé du dernier tableau, Les incendiaires est proposé en deux versions. Les deux derniers tableaux, celui de l'exécution et celui de l'inhumation "étant forcément très impressionnants, ne sont pas obligatoires " (p. 196).

Tous ces films sont diffusés, il faut le rappeler, pendant la période où les exécutions sont devenues rares, et où l'on semble s'acheminer vers l'abolition de la peine de mort. En 1908, année qui représente une sorte d'acmé du débat, deux films montrent encore la guillotine, mais cette fois sous une forme onirique ou hallucinatoire. Ce sont des films dont le remords est le ressort et dont Pierrot est le personnage principal. Pierrot, figure majeure de l'imaginaire de ce temps-là, est souvent associé au thème de la décollation et même à l'image de la guillotine. Dans Jalousie et ivresse de Pierrot (Pathé), Pierrot, après avoir violemment quitté Colombine et s'être enivré, est en proie à des hallucinations, puis s'endort sur un banc et rêve qu'il tue son amante; on l'emmène vers la guillotine; le couperet s'abat et Pierrot tombe du banc. Dans L'empreinte ou la main rouge, de Paul Henry Burguet, Pierrot (un Pierrot qui, cette fois, ne porte pas le costume traditionnel) a vendu son silence à un assassin, et il est en passe de laisser exécuter un innocent; miné par le remords, il a la vision d'une guillotine, qui se substitue à une danseuse lascive. Ces images dessinent encore les aspirations collectives, mais à travers des apparitions évanescentes.

Pourtant, cette année-là, l'abolition de la peine de mort est écartée, et Armand Fallières se résout à refuser des grâces. Le lundi 11 janvier 1909, Le Petit Journal titre "Quatre têtes tomberont ce matin à Béthune" et ajoute: "Cette fois, c'est décidé! La justice va suivre son cours et satisfaction va être donnée à l'opinion publique qui réclamait énergiquement l'exécution des bandits qui, pendant des années, désolèrent la région du Nord»(Anonyme 1909, p. 1). La bande de chauffeurs des frères Pollet, comme celle du film de Méliès, s'attaquait 
aux fermes isolées et en torturait les habitants pour les voler. Le Petit Journal indique que "de nombreux voyageurs débarquent par tous les trains pour assister à l'exécution». "Et qu'on ne s'étonne pas de cette affluence», dit le rédacteur, qui cite un témoin: "Il y aurait eu une émeute, si on n'avait pas exécuté Pollet et sa bande!»

Le lendemain paraît le récit des exécutions. Le bourreau a décidé au dernier moment d'installer la guillotine, non place Lamartine, mais aux abords de la prison. C'est une "énorme déception pour les curieux venus de partout» (Anonyme 1909a, p. 1) et qui avaient loué leur place à prix d'or. Néanmoins, le public, dont une bonne partie est ivre (les estaminets sont restés ouverts toute la nuit), exprime sa satisfaction, "joyeusement", d'abord, à la perspective des exécutions, puis «de façon cynique et écœurante", par des cris, des invectives, des applaudissements pendant qu'elles se déroulent. On sent presque passer ici le souffle des anciens massacres.

Les quatre têtes tombent. La dernière est celle d'Abel Pollet. "... le corps d'Abel Pollet n'a pas trouvé de place dans le panier, dans lequel il a roulé, et qui est plein. Le cou sectionné apparaît alors dans toute sa hideur. Deux flots de sang bouillonnant s'échappant en jets, viennent éclabousser les pieds des spectateurs horrifiés" (p. 2).

Si «horrifiés» qu’ils aient été, il ne semble pas que les spectateurs soient rassasiés par ce premier spectacle. Dès le lendemain, et les jours suivants, Le Petit Journal s'interroge sur l'identité du prochain décapité, avant de se demander le 19 janvier, avec une sorte de gourmandise: "Pour qui M. Deibler a-t-il aiguisé hier son couperet?»(Anonyme 1909b). La réponse tombe quelques jours plus tard: un certain Danvers doit être exécuté à Carpentras (Anonyme 1909c). Ce sera ensuite le tour de la bande des chauffeurs de la Drôme. En 1909 et dans les années suivantes, la guillotine fonctionne de nouveau régulièrement: dix condamnés sont exécutés, par exemple, en 1910 (Anonyme 1912, p. 28).

Or, pour la quadruple exécution de Béthune, le ministre de la Justice avait pris soin d'interdire l'usage de tout appareil cinématographique. Mais en 1908 est née la presse filmée avec Pathé Faits divers. Les opérateurs Pathé passent outre, semble-t-il, et 
filment ces authentiques décapitations, pérennisant ainsi ces visions fugitives de la mort vraie. Le ministre de la Justice saisit alors son collègue de l'Intérieur, qui adresse, le jour même, une circulaire télégraphique à tous les préfets, indiquant que, malgré l'interdiction:

...des clichés de cette scène auraient pu être pris par subterfuge ou surprise en vue de leur utilisation pour des spectacles cinématographiques. Il se pourrait également que dans [le] même but des industriels établissent des clichés purement imaginaires. J'estime qu'il est indispensable d'interdire tous spectacles cinématographiques publics de ce genre susceptibles de provoquer des manifestations troublant l'ordre et la tranquillité publics (Montagne 1997, p. 86).

Cette circulaire rédigée dans l'urgence est l'acte de naissance de la censure cinématographique en France ${ }^{4}$. On voit ce qu'il s'agit d'interdire: le spectacle de la mort réelle, et plus encore celui, inédit, de la décapitation vraie; est interdite aussi, dans cet élan, toute représentation de décapitation. Dans les années suivantes, ces interdits sont, semble-t-il, respectés. On ne voit plus tomber les têtes. Dans Cagliostro, de Camille de Morlhon et Gaston Velle (1910), lorsque Cagliostro révèle à MarieAntoinette ce que sera sa fin sur l'échafaud, on aperçoit seulement le début de la scène se profilant dans un flacon.

Le cas du premier épisode du Fantômas de Louis Feuillade, en 1913, est plus révélateur encore: il semble infliger délibérément une déception au spectateur-lecteur. Il s'intitule "À l'ombre de la guillotine». Dans le roman de Souvestre et Allain (1911, p. 414), Fantômas, arrêté et condamné à mort, réussit à faire décapiter à sa place un acteur grimé et drogué. Dans l'adaptation cinématographique, alors que la scène de l'exécution a été préparée de longue main, alors qu’on a déjà découpé le col du condamné, le stratagème est tout à coup découvert par Juve. On ne verra pas la guillotine fonctionner.

Si l'interdit porte alors aussi sur les reconstitutions, c'est qu'elles renverraient trop aisément, dans l'atmosphère tendue de ces années-là, au réel, et au spectacle du réel par excellence qu'est peut-être celui de la mort, et de la mort visible, certaine et instantanée qu'est la mort par décapitation. 
Mais ce qui est surtout en cause, ce sont les caractères mêmes du cinéma, sa puissance spectaculaire et son ambiguïté foncière, qui apparaissent nettement dans les années 1905-1909; le cinéma s'institutionnalise, sa fréquentation devient ordinaire, les actualités dites reconstituées cèdent la place à la presse filmée, qui s'érige en spectacle du réel, en spectacle, si l'on veut, à la fois médiat et immédiat. Médiat à cause de la machine et de la distance, immédiat parce que, tout de même, "cela a été». Le cinéma instaure, dans ces années-là, cette visibilité paradoxale, et il peut tout montrer, même ce qui apparait, de plus en plus, comme l'horreur.

Sous le circonstanciel, c'est un tabou qui est ici en cause, en effet, et c'est bien aussi ce qui fait la singularité du spectacle de la décapitation. En ce début de $\mathrm{XX}^{\mathrm{e}}$ siècle, on peut même dire que c'est plutôt un ensemble de tabous et de transgressions qu'il met en jeu: tabou de la vision du cadavre et de la mort donnée, tabou de la tête coupée, tabou de la guillotine. Ces thèmes se recouvrent partiellement, bien entendu, mais pas complètement, et leurs lignes d'évolution ne sont pas forcément les mêmes. Ils s'entrelacent ici en des figures complexes dont nous pouvons seulement esquisser l'analyse.

Le spectacle de la mort, la vision du cadavre ont fait l'objet d'une prohibition progressive dans les dernières décennies du $\mathrm{XIX}^{\mathrm{e}}$ siècle, mais il faut souligner la concomitance, dans les années 1907, 1908 et 1909, d'une part de l'interdiction de l'exposition à la Morgue, de la suspension des exécutions, suivie de leur spectaculaire reprise, du débat et du vote sur la publicité et sur l'abolition de la peine de mort, et d'autre part de l'institutionnalisation du cinéma et de l'établissement de la censure cinématographique dans le but d'empêcher la dissémination du spectacle des exécutions. On a été contraint, sous la pression de l'opinion, de maintenir la peine de mort et même de se résigner à sa publicité, tout en la rendant aussi peu effective que possible; il est évidemment exclu de l'amplifier infiniment et d'exacerber ses effets comme peut le faire le cinéma, universel colporteur d'images vraies. Le spectacle de la mort subsiste, mais le cinéma ne le montrera pas.

Mais il y a plus. L'exécution est décapitation. Or, si le bourreau ne présente plus la tête coupée à la foule, comme il le faisait sous la Terreur, le souvenir subsiste des anciennes monstrations. 
La culture du XIX ${ }^{e}$ siècle a été obsédée par la tête coupée; j'ai cité le cas de La femme au collier de velours, mais les occurrences littéraires ou iconographiques de têtes séparées du corps sont innombrables. La fin du siècle s'attache particulièrement à deux figures opposées dans lesquelles elle semble vouloir reconnaître ses doutes et ses angoisses: Judith et Salomé, femmes séductrices, fatales et coupeuses de têtes, tête de barbare ou tête de saint. La figure lascive et cruelle de Salomé, en particulier, à laquelle des centaines d'œuvres sont consacrées, est peut-être la figure fin de siècle par excellence, figure largement dépréciative par laquelle l'époque se laisse fasciner.

On peut sembler très loin, avec Gustave Moreau, Massenet ou Mallarmé, des vociférations de Béthune. On se souvient pourtant que dans L'empreinte, une danseuse qui pouvait être un avatar de Salomé se métamorphosait en guillotine. Il n'y avait là rien de fortuit, car la guillotine, Salomé moderne, géométrique et rigide, est alors féminisée et érotisée de façon parfaitement explicite. En argot, elle est "la veuve», mais aussi, simplement, "Madame» ou "Mademoiselle»; "épouser la veuve», c'est être guillotiné. De nombreux écrivains de la fin du XIX ${ }^{\mathrm{e}}$ siècle développent le thème d'une guillotine sexualisée. C'est le cas de Gustave Le Rouge, en 1893, dans une de ses premières nouvelles, "Notre-Dame la Guillotine». Gorgius, le "grand Répresseur», songe à la guillotine, qu'il utilise pour exterminer les «Pauvres»:

Elle se dressait en son imagination comme une idole embrumée de mystère $[\ldots]$, comme une attirante et traitresse femelle dont les jambes rigides, dont le sexe fallacieux incitaient l'humanité aux coïts monstrueux du cou et de la lunette (Le Rouge 1986, p. 1165).

Peu après, il fait monter chez lui une jeune fille. Il la possède, mais quand il veut s'arracher à son étreinte, il se sent paralysé comme l'homme que l'on jette entravé sur la planche de la guillotine. Il est tombé «dans les bras vengeurs de "Notre-Dame la Guillotine” "(p. 1168).

Et Jules Jouy (1888, p. 264-265), dans l'un des poèmes, intitulé La veuve, qu'il consacre à la décapitation, fait de l'exécution une nuit de noces: 
Elle ne sort de sa maison

Que lorsqu'il faut qu'un bandit meure.

Dans sa voiture de gala

Qu'accompagne la populace,

Elle se rend, non loin de là,

Et triste descend sur la place.

[...]

Les témoins, le prêtre et la loi,

Voyez, tout est prêt pour la noce.

[...]

Alors, tendant ses longs bras roux,

Bichonnée, ayant fait peau neuve,

Elle attend son nouvel époux

[...]

Pendant que la foule, autour d'eux,

Regarde, frissonnante et pâle

Dans un accouplement hideux,

L'homme crache son dernier râle.

Si l'exécution est un acte sexuel, si la lunette est un sexe féminin, la décapitation est évidemment une castration. L'imaginaire fin de siècle n'en fait en quelque sorte aucun mystère. Ce n'est sans doute pas la raison pour laquelle Freud trouve, en 1922, l'interprétation de la figure de Méduse «à portée de main »:

Nous n'avons pas souvent tenté l'interprétation de figures mythologiques prises individuellement. Elle est, pour la tête coupée de Méduse éveillant l'horreur, à portée de main.

Décapiter $=$ castrer. L'effroi de la Méduse est donc effroi de castration, qui est rattaché à la vue de quelque chose. À partir de nombreuses analyses nous connaissons cette occasion, elle se produit lorsque le garçon, qui jusque-là ne voulait pas croire à la menace, vient à voir un organe génital féminin. Vraisemblablement un organe adulte, ourlé de cheveux, au fond celui de la mère (Freud 1991, p. 163).

La vision de la décapitation est ainsi vision d'horreur pour une série de raisons concourantes: elle est vision de l'horreur de la mort, simplement; sa fulgurance suscite la pensée d'une tête percevant l'horreur de sa propre section; elle ravive le souvenir de l'horreur d'anciennes tueries; enfin, elle renvoie à l'horreur d'angoisses archaïques suscitées par un voir. Cet effroi, la foule 
sadique le soutient, cependant. C'est l'autre qui meurt, qu'on décapite ou qu'on castre. On se souvient, dans le poème de Jules Jouy, de la foule "frissonnante et pâle» regardant "l'accouplement hideux ".

Pourtant, ce qui subsiste du spectacle de la décapitation réelle est un vestige. Une très petite partie du public, sélectionnée, y assiste effectivement. Le gros de la foule est tenu à l'écart. Une page a été tournée dans l'histoire du spectacle de la mort au début $\mathrm{du} \mathrm{XX}^{\mathrm{e}}$ siècle. Le cinéma, grand dispositif voyeuriste, ne montrera pas ce qui ne doit plus être vu, et ce tabou sera rarement transgressé. Il s'institue, en somme, de renoncer à une partie de son pouvoir, qui est de tout montrer. Et s'il est vrai que le cinéma s'est frayé un "chemin vers ce visible absolu qu'est la visibilité de l'horreur - le film par excellence étant le film d'horreur" (Kristeva 1998, p. 137), cela n'a pu être, évidemment, qu'à travers la médiation de l'artifice et de la fiction. Et tout se passe comme si ce qui ne devait plus tomber sous les regards pouvait se déployer dans l'imaginaire, en particulier par le truchement du cinéma, comme si le cinéma intervenait à propos, à la fin du XIX ${ }^{e}$ siècle, pour susciter, non les visions désormais interdites, mais leur reflet, non la tête de Méduse, mais son image dans le bouclier.

Et il y a en effet d'emblée quelque chose comme de l'horrifique disséminé dans tout le premier cinéma, mais de façon significativement diffuse et indécise. Michel Marie (1988, p. 65) a bien montré que le cinéma des origines «avait su donner libre cours aux fantasmes originaires les plus archaïques». Désir de voir le sexe de la mère, angoisse pour le voyeur d'être puni de mort, fantasmes de dévoration s'y lisent en effet sans qu'il soit nécessaire de forcer l'interprétation. Le voyeurisme sadique trouve aussi à s'y satisfaire. Qu'on parcoure par exemple le catalogue Pathé. Dans Jalousie et folie (1907), un fou mange des rats dans une cave, avant de devenir meurtrier. Dans Les chauffeurs (1907), un paludier est supplicié et meurt dans des contorsions d'agonie. Dans L'assassinat du Duc de Guise (1908), le corps du duc est jeté dans le feu. Dans La peur (1909), l'assassin rendu fou par la pensée de son crime, poignarde sa sœur en qui il croit revoir sa victime. Ce ne sont que quelques 
exemples de films dramatiques. Mais on a pu parler aussi à bon droit d' "horreur comique" (Michaud et Ribadeau Dumas 2004), et s'il y a quelques films d'exécutions, il y a d'innombrables décapitations comiques: tête qu'on coupe pour la raser (Le barbier fin-de-siècle, Pathé, 1896), qu'on arrache pour en soigner les dents (Chez le dentiste, Pathé, 1901), tête gonflée jusqu'à exploser (L’homme à la tête en caoutchouc, Méliès, 1901), têtes coupées multipliées (Méliès encore, Un homme de têtes, 1898, Le mélomane, 1903), etc. Il y a aussi de très nombreux démembrements, et le corps comique est à vrai dire soumis à toutes les tortures, à toutes les dégradations.

Ces motifs, supplices atroces, mutilations, cette folie sadique, ne sont pas propres au cinéma. Dans le temps même de la progressive occultation du spectacle de la mort, les romans policiers, les quotidiens et leurs suppléments illustrés, les magazines spécialisés dans les récits de faits divers, le théâtre même, et singulièrement ce théâtre spécialisé dans la perversité qu'est le Grand-Guignol, s'en étaient déjà emparés, avec une intensité croissante et d'une manière qui faisait la part de plus en plus belle à l'imaginaire (même quand il s'agissait de faits donnés pour authentiques), au visuel, à l'effet de réel. C'est là, à travers ces différents supports textuels et iconiques, qu'émergeait sans doute ce que nous appelons «horreur» (au sens esthétique, bien entendu). Mais sur ce terrain, le cinéma était évidemment incomparable. Il était l'outil idéal. Il y eut ainsi d'emblée une horreur cinématographique, ambiguë, d'abord, ignorante d'ellemême; mais la fidélité du cinéma au réel lui fit rencontrer l'interdit qui, peut-être, lui permettrait de fixer ses limites et de se spécifier. Le paradoxe, c'est que cela ne put advenir vraiment qu'après le violent retour des visions refoulées qu'allaient provoquer la Grande Guerre et la grippe espagnole.

Centre national de la recherche scientifique (France)

\section{NOTES}

1. Un examen de cette question complexe excéderait le cadre de cet article. Je ne peux que renvoyer aux travaux de Noël Carroll (1990), de Carol J. Clover (1992) et d'Éric Dufour (2006). 
2. La Morgue reçoit 989 "cadavres» (y compris les foetus et les débris humains) en 1892, et 909 en 1893, par exemple (archives de la Préfecture de police, DA 34 Morgue).

3. Voir notamment, à ce sujet: Dominguez Leiva 2004, Gerould 1992, Janes 2005 et Lasowski 2007.

4. Il va sans dire que la censure a pu, en d'autres lieux, surgir de circonstances différentes. Voir, par exemple, pour les États-Unis: Grieveson 2004.

\section{RÉFÉRENCES BIBLIOGRAPHIQUES}

Anonyme 1989: Anonyme, Compte général de l'administration de la justice criminelle en France pendant l'année 1880 et rapport relatif aux années 1826 à 1880, publié et commenté par Michelle Perrot et Philippe Robert, Genève/Paris, Slatkine Reprints, 1989.

Anonyme 1900 : Anonyme, Compte général de l'administration de la justice criminelle en France pendant l'année 1897, Paris, Imprimerie nationale, 1900.

Anonyme 1903: Anonyme, Compte général de l'administration de la justice criminelle en France pendant l'année 1901, Paris, Imprimerie nationale, 1903.

Anonyme 1903a: Anonyme, Délibérations du conseil municipal de Paris, année 1902, Paris, Imprimerie nationale, 1903.

Anonyme 1908: Anonyme, Compte général de l'administration de la justice criminelle en France pendant l'année 1906, Paris, Imprimerie nationale, 1908.

Anonyme 1909: Anonyme, "Quatre têtes tomberont ce matin à Béthune», Le Petit Journal, 11 janvier 1909, p. 1.

Anonyme 1909a: Anonyme, "La quadruple exécution de Béthune», Le Petit Journal, 12 janvier 1909, p. 1-2.

Anonyme 1909b: Anonyme, "Pour qui M. Deibler a-t-il aiguisé hier son couperet?", Le Petit Journal, 19 janvier 1909, p. 1.

Anonyme 1909c: Anonyme, "La guillotine fonctionnera après-demain ", Le Petit Journal, 24 janvier 1909, p. 1.

Anonyme 1912: Anonyme, Compte général de l'administration de la justice criminelle en France pendant l'année 1910, Paris, Imprimerie nationale, 1912.

Ariès 1977: Philippe Ariès, L'homme devant la mort, II. La mort ensauvagée, Paris, Seuil, 1977.

Bertherat 2003: Bruno Bertherat, La Morgue de Paris au XIX siècle (1804-1907). Les origines de l'institut médico-légal ou Les métamorphoses de la machine, thèse de doctorat, Université Paris 1, 2003.

Borel 1843: Petrus Borel, "Gottfried Wolfgang", Revue parisienne (La Sylphide), $4^{\mathrm{c}}$ série, tome VIII, 1843, p. 331-334.

Carroll 1990 : Noël Carroll, The Philosophy of Horror, or Paradoxes of the Heart, New York, Routledge, 1990.

Cherbuliez 1891 : Ernest Cherbuliez, "La Morgue de Paris», La Revue des deux mondes, janvier-février 1891, p. 344-381.

Clover 1992: Carol J. Clover, Men, Women, and Chainsaws. Gender in the Modern Horror Film, London, British Film Institute, 1992.

Corbin 2005: Alain Corbin (dir.), Histoire du corps. Tome 2. De la Révolution à la Grande Guerre, Paris, Seuil, 2005.

Dechambre 1869: Amédée Dechambre (dir.), Dictionnaire encyclopédique des sciences médicales, série 1, tome 3, 1869.

Spectacles douloureux, exhibitions malsaines. Présentations et représentations de la mort à Paris au XIX siècle 
Deschamps 1897 : Émile Deschamps, "À la morgue», Carnet d'un voyageur. Au harem, notes, croquis, souvenirs, impressions, Paris, Société libre d'édition des gens de lettres, 1897, p. 163-185.

Dominguez Leiva 2004: Antonio Dominguez Leiva, Décapitations. Du culte des crânes au cinéma gore, Paris, Presses universitaires de France, 2004.

Dufour 2006: Éric Dufour, Le cinéma d'horreur et ses figures, Paris, Presses universitaires de France, 2006.

Dumas 2006: Alexandre Dumas, Les mille et un fantômes, précédé de La Femme au collier de velours [1849], Paris, Gallimard, 2006.

Edelman 2006 : Nicole Edelman, Histoire de la voyance et du paranormal. Du XVIIf siècle à nos jours, Paris, Seuil, 2006.

Flaubert 1980 : Gustave Flaubert, Correspondance, tome II, Paris, Gallimard/ Bibliothèque de la Pléiade, 1980.

Freud 1991: Sigmund Freud, "La tête de Méduse», Euvres complètes. Psychanalyse. Vol. XVI: 1921-1923, 2 édition, Paris, Presses universitaires de France, 1991, p. 161164.

Gerould 1992: Daniel Gerould, Guillotine. Its Legend and Lore, New York, Blast Books, 1992.

Grieveson 2004: Lee Grieveson, Policing Cinema. Movies and Censorship in EarlyTwentieth-Century America, Berkeley/Los Angeles/London, University of California Press, 2004.

Guillot 1887: Adolphe Guillot, Paris qui souffre. La basse geôle du Grand Châtelet et les morgues modernes, Paris, Pierre Rouquette, 1887.

Heine 1878 : Heinrich Heine, De la France, Paris, Michel Lévy frères, 1878.

Imbert 1998: Jean Imbert, La peine de mort, Paris, Presses universitaires de France, 1998.

Irving 1951: Washington Irving, "L'Aventure d'un étudiant allemand» [1824], Lî̀le fantôme et autres contes, Paris, Robert Marin, 1951, p. 83-97.

Janes 2005: Regina Janes, Losing our Heads. Beheadings in Literature and Culture, New York/London, New York University Press, 2005.

Janin 1842: Jules Janin, L'âne mort et la femme guillotinée [1829], Paris, Ernest Bourdin, 1842 .

Jouy 1888 : Jules Jouy, Les chansons de l'année, Paris, Bourbier et Lamoureux, 1888.

Kristeva 1998 : Julia Kristeva, Visions capitales, Paris, Réunion des musées nationaux, 1998.

Lasowski 2007 : Patrick Wald Lasowski, Guillotinez-moi! Précis de décapitation, Paris, Le Promeneur, 2007.

Le Rouge 1986: Gustave Le Rouge, "Notre-Dame la Guillotine» [1893], Le mystérieux docteur Cornélius..., Paris, Robert Laffont, 1986, p. 1164-1168.

Leroux 1924: Gaston Leroux, "La femme au collier de velours", Aventures incroyables, Paris, Robert Laffont, 1924, p. 876-887.

Malthête et Mannoni 2008 : Jacques Malthête et Laurent Mannoni, L'œuvre de Georges Méliès, Paris, La Martinière/Cinémathèque française, 2008.

Marie 1988 : Michel Marie, "La scène des fantasmes originaires", dans André Gaudreault (dir.), Ce que je vois de mon ciné: la représentation du regard dans le cinéma des premiers temps, Paris, Méridiens-Klincksieck, 1988, p. 61-65.

Michaud et Ribadeau Dumas 2004: Philippe-Alain Michaud et Isabelle Ribadeau Dumas (dir.), L'horreur comique. Esthétique du slapstick, Paris, Centre Pompidou, 2004. 
Monnier 1984: Henri Monnier, Scènes populaires. Les bas-fonds de la société, Paris, Gallimard, 1984.

Montagne 1997 : Albert Montagne, «Droit et libertés publiques. Les actualités filmées ont enfanté la censure du cinéma français en 1909", Les Cahiers de la Cinémathèque, $\mathrm{n}^{\circ}$ 66, 1997, p. 83-89.

Rollinat 1885 : Maurice Rollinat, Les névroses, Paris, G. Charpentier, 1885.

Souvestre et Allain 1911: Pierre Souvestre et Marcel Allain, Fantômas, Paris, Arthème Fayard, 1911.

Van Gennep 1998: Arnold Van Gennep, Le folklore français. Du berceau à la tombe, cycles de carnaval - Carême et de Pâques, Paris, Robert Laffont, 1998.

Vovelle 1983: Michel Vovelle, La mort en Occident de 1300 à nos jours, Paris, Gallimard, 1983.

\section{ABSTRACT}

\section{Painful Spectacles, Unhealthy Displays:} The Presentation and Representation of Death in Nineteenth-Century Paris

\section{Patrick Désile}

Taking the view that the central theme of horror films is death, this article enquires into the context of their emergence by examining the spectacles, in France, which predated them and whose subject was death. In addition to countless representations of death, the nineteenth century was a time of presentations of death. Public displays at the Paris morgue and public executions were attended by many, and narratives accompanied these showings. Horror films, however, are not their continuation by other means. The author describes a more complex process in which our view of death changes over the course of the century. Beginning in the 1870s, a process of concealment began to take hold. Public displays at the morgue ceased in 1907, and the first bill to abolish the death penalty was introduced in 1908. During the same period, however, films showed execution scenes. When decapitations, which had been suspended for a few years, recommenced in 1909, it appears they were filmed. But any screening of executions was quickly prohibited, thereby giving birth to film censorship in France. The spectacle of real decapitation was henceforth taboo in French cinema. For a variety of reasons, decapitation might be viewed as the horror spectacle par excellence, as the author's discussion of imaginary depictions of it reveals. 